\title{
Monohydroperoxides Formed by Autoxidation and Photo- sensitized Oxidation of Methyl Eicosapentaenoate
}

\author{
Ryo Yamauchi, Tomoo Yamada, Koji Kato \\ and Yoshimitsu UENO \\ Department of Agricultural Chemistry, Gifu University, \\ Gifu 501-11, Japan
}

Received July 27, 1983

\begin{abstract}
Methyl eicosapentaenoate (methyl 5,8,11,14,17-eicosapentaenoate) was subjected to autoxidation and methylene blue sensitized photooxidation. Methyl eicosapentaenoate monohydroperoxides, the primary products of the autoxidation and photosensitized oxidation, were isolated by silica gel column chromatography, and characterized by ultraviolet, infrared and nuclear magnetic resonance spectra. The isomeric composition of the monohydroperoxides were determined by gas chromatography-mass spectrometry as follows: the 5-, 8-, 9-, 11-, 12-, 14-, 15- and 18-isomers (autoxidation), and the 5-, 6-, 8-, 9-, 11-, 12-, 14-, 15-, 17- and 18-isomers (photosensitized oxidation). Methyl eicosapentaenoate was readily oxidized both by autoxidation and by photosensitized oxidation.
\end{abstract}

Unsaturated lipids are subjected to lipid peroxidation which leads to nutritional damage in foods and physiological damage in living organisms. The oxidation mechanisms of unsaturated lipids by the free radical reaction and by the singlet molecular oxygen $\left({ }^{1} \mathrm{O}_{2}\right)$ have been investigated. $\left.{ }^{1 \sim 3}\right)$ Polyunsaturated fatty acids consisting of triacylglycerols and phospholipids produce positional isomers of monohydroperoxide as the primary oxidation products.

Eicosapentaenoic acid $(5,8,11,14,17$-eicosapentaenoic acid) has been reported to contribute to a low incidence of acute myocardial infarction and atherosclerosis as well as to the bleeding tendency. ${ }^{4,5)}$ Eicosapentaenoic acid is considered to be susceptible to lipid peroxidation because of the presence of five double bonds in the molecule. Fukuzumi et $\left.a{ }^{6}{ }^{6}\right)$ reported the peroxide concentrates from autoxidized methyl eicosapentaenoate, but they could not determine the structures of the peroxides. Noble and Nawar ${ }^{7,8)}$ reported the volatile decomposition products of methyl docosahexaenoate after autoxidation, and proposed mechanisms for the formation of the volatile compounds. However, little is known about the peroxidation mechanism of eicosapentaenoic acid.

This paper reports the autoxidation and the ${ }^{1} \mathrm{O}_{2}$ oxidation of methyl eicosapentaenoate. Methylene blue sensitized photooxidation was employed as the ${ }^{1} \mathrm{O}_{2}$ generating system. ${ }^{9)}$ The monohydroperoxides were isolated, and their structures are characterized. The rate of peroxidation of methyl eicosapentaenoate is compared with those of the other unsaturated fatty acid methyl esters.

\section{MATERIALS AND METHODS}

Materials. Methyl eicosapentaenoate concentrate was kindly provided by Taiyo Fishery Co., Ltd. Methyl eicosapentaenoate was purified by reverse-phase high performance liquid chromatography (HPLC) on a column $(4.6 \times$ $250 \mathrm{~mm}$ ) of LiChrosorb RP-18 (Merck, $10 \mu \mathrm{m}$ in particle size) using methanol-water $(95: 5, \mathrm{v} / \mathrm{v})$ as the solvent. The purity of the methyl eicosapentaenoate was $>99 \%$ by gas liquid chromatography. Methyl linoleate and methyl linolenate were purchased from Tokyo Kasei Co., and methyl arachidonate was prepared from pig liver phospholipids. ${ }^{11)}$ These methyl esters were also purified by HPLC as described above. Other reagents used were of analytical grade.

Peroxidation procedure. Methyl eicosapentaenoate 
$(300 \mathrm{mg})$ in a glass vial was autoxidized at $37^{\circ} \mathrm{C}$ with bubbling air for $20 \mathrm{hr}$. The rate of autoxidation of methyl eicosapentaenoate, methyl arachidonate, methyl linolenate and methyl linoleate was determined in a thin lipid film. $50 \mu \mathrm{l}$ of $10^{-2} \mathrm{M}$ methyl ester in ethanol solution was added to a test tube $(1.5 \mathrm{~cm}$ in diameter), and then the solvent was removed by evaporating in vacuo to form a thin lipid film. The sample was placed in an incubator maintained at $37^{\circ} \mathrm{C}$ in the dark. At intervals, each sample was dissolved in $5 \mathrm{ml}$ of ethanol, and the amount of conjugated diene was measured at $235 \mathrm{~nm}$.

Methyl eicosapentaenoate was photo-oxidized in an ethanol solution containing methylene blue as the photosensitizer. Methyl eicosapentaenoate $(150 \mathrm{mg})$ was dissolved in $50 \mathrm{ml}$ of ethanol containing methylene blue $(2 \mathrm{mg})$. The solution in a reaction vessel was placed in a water bath at $25^{\circ} \mathrm{C}$, and shaken mechanically. The light source was a $60 \mathrm{~W}$ tungsten projection lamp at a distance of $20 \mathrm{~cm}$ from the vessel. The reaction was carried out for $6 \mathrm{hr}$. The rate of photosensitized oxidation of methyl eicosapentaenoate, methyl arachidonate, methyl linolenate and methyl linoleate was determined in an ethanol solution. $10^{-2} \mathrm{M}$ methyl ester in an ethanol solution containing $10^{-4} \mathrm{M}$ methylene blue was added to a reaction vessel, and irradiated with a $60 \mathrm{~W}$ lamp at $25^{\circ} \mathrm{C}$. At intervals, $50 \mu \mathrm{l}$ of the sample was dissolved in $5 \mathrm{ml}$ of ethanol, and measured at $235 \mathrm{~nm}$.

Preparation and characterization of monohydroperoxides. The peroxidized methyl eicosapentaenoate was analyzed by thin layer chromatography (TLC), using a Merck pre-coated silica gel 60 plate, with hexane-ethyl ether-acetic acid $(60: 40: 1, v / v)$. Lipids were detected with $50 \% \mathrm{H}_{2} \mathrm{SO}_{4}$ in saturated $\mathrm{K}_{2} \mathrm{CrO}_{7}$ solution, and peroxides were detected with $5 \% \mathrm{KI}$ in methylcellosolve solution. Monohydroperoxides were separated by silica gel column chromatography with stepwise elutions of 5 and $30 \%$ ethyl ether in hexane. The unoxidized lipid was eluted with the $5 \%$ ethyl ether fraction, and monohydroperoxide eluted with the $30 \%$ ethyl ether fraction.

The isolated monohydroperoxides were characterized by ultraviolet (UV) spectra, infrared (IR) spectra and nuclear magnetic resonance (NMR) spectra. The NMR spectra were recorded at $90 \mathrm{MHz}$ with a Hitachi R-22 spectrometer for solutions in $\mathrm{CCl}_{4}$, tetramethylsilane being used as the internal standard. The monohydroperoxides were reduced by $\mathrm{NaBH}_{4}$ or hydrogenated by a stream of hydrogen gas according to the method described by Terao and Matsushita. ${ }^{11)}$ The reduced or hydrogenated product was converted to a trimethylsilyl (TMS) derivative and analyzed by gas chromatography-mass spectrometry (GC-MS). GC-MS was carried out on a Hitachi M-52 apparatus. Operational conditions were the same as described previously. ${ }^{12)}$

\section{RESULTS}

\section{Autoxidation of methyl eicosapentaenoate}

Methyl eicosapentaenoate was autoxidized for $20 \mathrm{hr}$ and the oxidation products were analyzed by TLC. Figure 1 shows the densitometric pattern of autoxidized methyl eicosapentaenoate on TLC. Methyl eicosapentaenoate and many products were observed on the plate. The oxidation products were shown to be peroxides by the KI test. ${ }^{13)}$ The most mobile oxidation product, $\mathbf{A}$, was isolated by silica gel column chromatography. The UV spectrum of compound $\mathbf{A}$ gave an absorption maximum at $235.5 \mathrm{~nm}$ in ethanol $(\varepsilon=23,000)$ due to conjugated diene. The IR spectrum of compound $\mathbf{A}$ gave a strong absorption at $3400 \mathrm{~cm}^{-1}$, attributed to the hydroperoxy group. ${ }^{6)}$ Furthermore, the NMR spectrum of compound A showed a broad signal downfield, $\delta c a .8 .5 \mathrm{ppm}$, as a result of the hydroperoxy proton. ${ }^{14)}$ After the addition of $\mathrm{D}_{2} \mathrm{O}$ to the NMR tube, the signal diminished to the base line. The proton number in the region of the hydroperoxy proton calculated from an integral based on the terminal methyl proton $(\delta$ $0.9 \sim 1.1$ ) was 0.9 . Thus, compound $\mathbf{A}$ was identified to be methyl eicosapentaenoate monohydroperoxide.

The TMS derivative of $\mathrm{NaBH}_{4}$-reduced

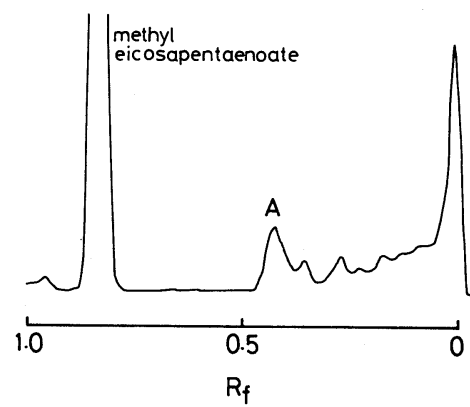

FIG. 1. Densitometric Pattern of Autoxidized Methyl Eicosapentaenoate on the TLC Plate.

An aliquot of the autoxidized methyl eicosapentaenoate $\left(37^{\circ} \mathrm{C}\right.$ for $20 \mathrm{hr}$ ) was spotted on a TLC plate. The plate was developed with a solvent of hexane-ethyl ether-acetic acid (60: $40: 1, \mathrm{v} / \mathrm{v})$. After it had been sprayed with $50 \% \mathrm{H}_{2} \mathrm{SO}_{4}$ in saturated $\mathrm{K}_{2} \mathrm{CrO}_{7}$ solution, the plate was heated for 10 $\mathrm{min}$ and monitored by a densitometer at $350 \mathrm{~nm}$. 


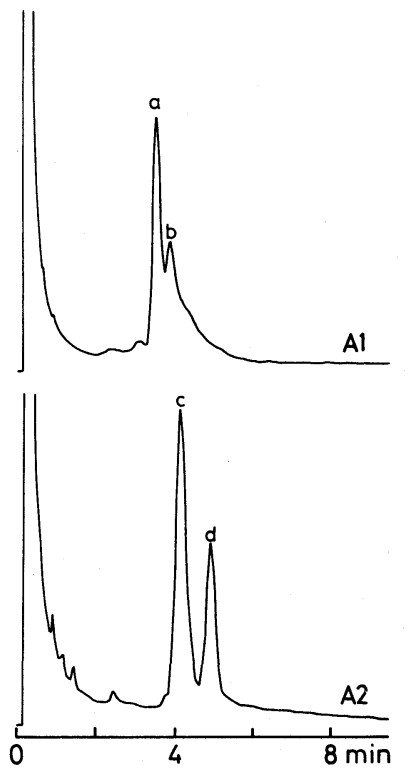

FIG. 2. Gas Chromatograms of the TMS Derivatives of Monohydroperoxide Formed by the Autoxidation of Methyl Eicosapentaenoate after $\mathrm{NaBH}_{4}$ Reduction (A1) and Hydrogenation (A2).

compound A was analyzed by GC-MS (Fig. 2A1). Two vicinal peaks, a and b, appeared on the chromatogram. The mass spectra of peaks $\mathrm{a}$ and $\mathrm{b}$ were almost the same. The presence of the characteristic ions at $m / z 404\left(\mathrm{M}^{+}\right), 389$ $\left(\left[\mathrm{M}-\mathrm{CH}_{3}\right]^{+}\right)$and $314\left([\mathrm{M}-90]^{+}\right.$, loss of trimethylsilanol) indicated a hydroxy methyl eicosapentaenoate TMS derivative. When the double bonds were saturated by hydrogenation, the TMS derivative showed two peaks, $c$ and $d$, on the chromatogram (Fig. 2-A2). From the mass spectra, peaks $\mathrm{c}$ and $\mathrm{d}$ were identified to be hydroxy methyl eicosanoate TMS derivatives $;{ }^{15)} \mathrm{m} / z 399\left(\left[\mathrm{M}-\mathrm{CH}_{3}\right]^{+}\right), 383$ $\left(\left[\mathrm{M}-\mathrm{CH}_{3} \mathrm{O}\right]^{+}\right)$and $367 \quad\left(\left[\mathrm{M}-\left(\mathrm{CH}_{3}+\right.\right.\right.$ $\left.\mathrm{CH}_{3} \mathrm{OH}\right]^{+}$). Characteristic fragmentation patterns indicated that peak $\mathrm{c}$ was a mixture of C-5 (m/z 203 and 313), C-8 $(m / z 245$ and 271), C-9 ( $m / z 257$ and 259), C-11 ( $m / z 229$ and 287), C-12 ( $m / z 215$ and 301), C-14 ( $m / z 187$ and 329) and $\mathrm{C}-15(\mathrm{~m} / \mathrm{z} 173$ and 343), and peak d was C-18 (m/z 131 and 385) positional isomers of the TMS group. From the above spectral data, compound $\mathbf{A}$ was identified to be isomeric methyl eicosapentaenoate mono-

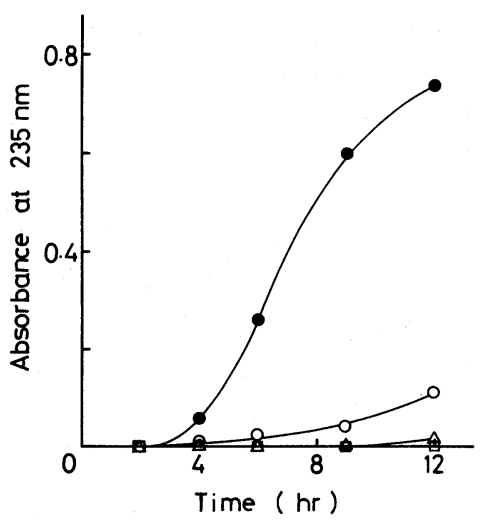

FIG. 3. Time Course of Autoxidized Methyl Eicosapentaenoate, Methyl Arachidonate, Methyl Linolenate and Methyl Linoleate.

The neat fatty acid methyl esters were autoxidized at $37^{\circ} \mathrm{C}$. - - methyl eicosapentaenoate; $\bigcirc-\bigcirc$, methyl arachidonate; $\triangle-\triangle$, methyl linolenate; $\square-\square$, methyl linoleate.

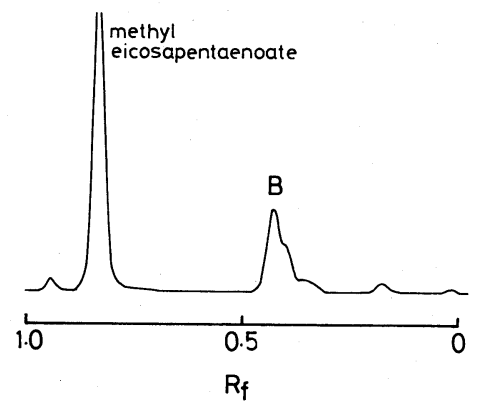

FIG. 4. Densitometric Pattern of the Photosensitized Oxidation of Methyl Eicosapentaenoate on the TLC Plate. An aliquot of the photosensitized oxidation products of methyl eicosapentaenoate $\left(25^{\circ} \mathrm{C}\right.$ for $\left.6 \mathrm{hr}\right)$ was spotted on a TLC plate. The conditions of TLC separation and detection were the same as described in Fig. 1.

hydroperoxides.

Figure 3 shows the rate of autoxidation of the methyl eicosapentaenoate, methyl arachidonate, methyl linolenate and methyl linoleate thin films at $37^{\circ} \mathrm{C}$. Methyl eicosapentaenoate was rapidly autoxidized. On the other hand, the other unsaturated esters scarcely oxidized during this incubation time.

Photosensitized oxidation of methyl eicosapentaenoate

Methyl eicosapentaenoate was photo- 
oxidized for $6 \mathrm{hr}$ in an ethanol solution containing methylene blue as the photosensitizer. Figure 4 shows the densitometric pattern of photo-oxidized methyl eicosapentaenoate on TLC. Two spots corresponding to methyl eicosapentaenoate and an oxidation product, $\mathbf{B}$, appeared on the plate. Compound $\mathbf{B}$ was positive to the KI test, and showed an absorption maximum at $235.5 \mathrm{~nm}$ in ethanol $(\varepsilon=$ 20,000). The IR spectrum gave a strong absorption at $3400 \mathrm{~cm}^{-1}$. The NMR spectrum showed a broad hydroperoxy proton signal at $\delta c a .8 .5 \mathrm{ppm}$ and the number of the hydroperoxy proton was 1.1. Thus, compound $\mathbf{B}$ was identified to be methyl eicosapentaenoate monohydroperoxide.

The TMS derivative of $\mathrm{NaBH}_{4}$-reduced compound $\mathbf{B}$ was analyzed by GC-MS (Fig. 5B1). Two vicinal peaks, $a$ and $b$, appeared on the chromatogram. The mass spectra of the two peaks, showing almost the same result as described in the TMS derivative of $\mathrm{NaBH}_{4}$ reduced compound $\mathbf{A}$, indicated a hydroxy methyl eicosapentaenoate TMS derivative. When the TMS derivative of hydrogenated compound B was analyzed by GC-MS, three peaks, c, d and e, appeared on the chromatogram (Fig. 5-B2). From the mass spectra, peaks $\mathrm{c}, \mathrm{d}$ and e were identified to be hydroxy methyl eicosanoate TMS derivatives; $\mathrm{m} / z 399$ $\left(\left[\mathrm{M}-\mathrm{CH}_{3}\right]^{+}\right), 383\left(\left[\mathrm{M}-\mathrm{CH}_{3} \mathrm{O}\right]^{+}\right)$and 367 $\left(\left[\mathrm{M}-\left(\mathrm{CH}_{3}+\mathrm{CH}_{3} \mathrm{OH}\right]^{+}\right)\right.$. Characteristic fragmentation patterns indicated that peak $\mathrm{c}$ was a mixture of C-5 $(\mathrm{m} / \mathrm{z} 203$ and 313), C-6 $(\mathrm{m} / z 217$ and 299), C-8 ( $m / z 245$ and 271), C-9 ( $m / z 257$ and 259), C-11 ( $m / z 229$ and 287), C-12 (m/z 215 and 301), C-14 ( $m / z 187$ and 329) and C-15 ( $m / z 173$ and 343), peak d was C-17 ( $m / z 145$ and 371), and peak e was C-18 $(\mathrm{m} / \mathrm{z} 131$ and 385) positional isomers of the TMS group. From the above data, compound $\mathbf{B}$ was identified to be isomeric methyl eicosapentaenoate monohydroperoxides.

Figure 6 shows the rate of methylene blue sensitized photooxidation of methyl eicosapentaenoate, methyl arachidonate, methyl linolenate and methyl linoleate in ethanol solutions. The formations of the conjugated

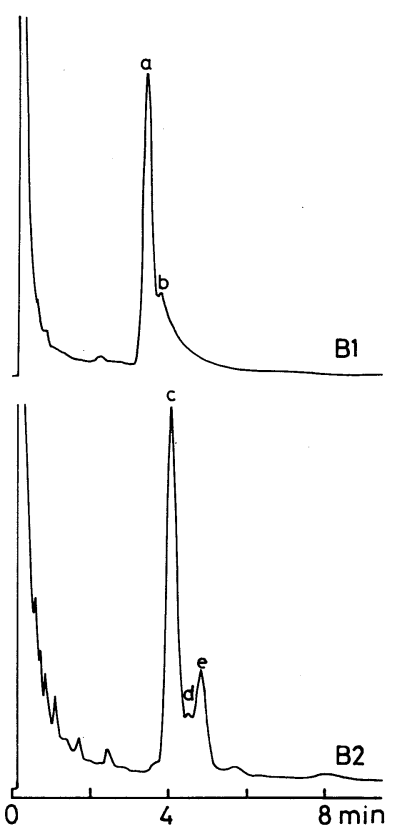

Fig. 5. Gas Chromatograms of the TMS Derivatives of Monohydroperoxide Formed by the Photosensitized Oxidation of Methyl Eicosapentaenoate after $\mathrm{NaBH}_{4}$ Reduction (B1) and Hydrogenation (B2).

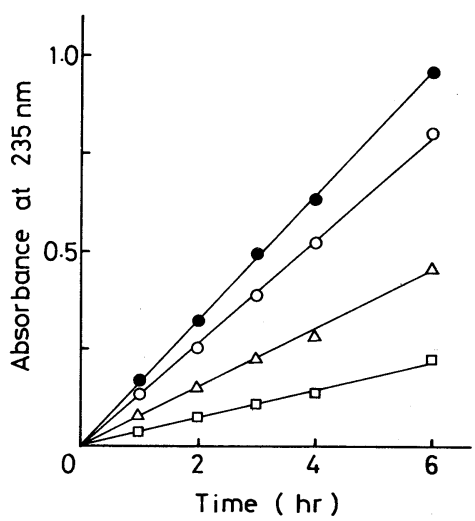

FIG. 6. Time Course of Photosensitized Oxidation of Methyl Eicosapentaenoate, Methyl Arachidonate, Methyl Linolenate and Methyl Linoleate.

The reaction mixture containing $10^{-2} \mathrm{M}$ fatty acid methyl ester and $10^{-4} \mathrm{M}$ methylene blue in ethanol solution was exposed to irradiation at $25^{\circ} \mathrm{C}$. - methyl eicosapentaenoate; $\bigcirc-\mathrm{O}$, methyl arachidonate; $\triangle-\triangle$, methyl linolenate; $\square-\square$, methyl linoleate.

diene were linear with the irradiation time. Methyl eicosapentaenoate showed the greatest formation of conjugated diene of the four 
unsaturated esters.

\section{DISCUSSION}

The primary oxygenated product of unsaturated lipids is monohydroperoxide. Many workers have reported the autocatalytic oxidation of oleate, linoleate, linolenate and arachidonate, and have characterized monohydroperoxide isomers ${ }^{11,16 \sim 20)}$ and their further oxygenated compounds. ${ }^{11,21 \sim 23)}$ When methyl eicosapentaenoate was autoxidized, the eight regioisomers of monohydroperoxide, that is, the 5-, 8-, 9-, 11-, 12-, 14-, 15- and 18isomers, were formed as the result of $\mathrm{O}_{2}$ attack on the pentadiene radicals. ${ }^{1,2)}$ Further oxygenated compounds were also detected on the TLC plate (Fig. 1). However, their structures are still uncertain. Arachidonic acid is the precusor of dienoic prostaglandins, ${ }^{24)}$ and eicosapentaenoic acid is the precusor of trienoic prostaglandins. ${ }^{25)}$ The nonenzymatic peroxidation of arachidonic acid has been reported to form prostaglandinlike compounds. ${ }^{26)}$ Therefore, the autoxidation of eicosapentaenoic acid may form prostaglandin-like compounds.

The lipid peroxidation is also caused by ${ }^{1} \mathrm{O}_{2}$ which can be produced by photosensitized oxidation. ${ }^{27)}$ The isomeric compositions of monohydroperoxides formed by the ${ }^{1} \mathrm{O}_{2}$ oxidation of unsaturated lipids have been investigated. ${ }^{15,28,29)}$ Singlet molecular oxygen reacts directly with double bonds by concerted addition, the so-called "ene type" reaction. ${ }^{1,2)}$ The ten regioisomers of monohydroperoxide, that is, 5-, 8-, 9-, 11-, 12-, 14-, 15- and 18isomers (conjugated isomer), and 6- and 17isomers (nonconjugated isomer), were formed from methyl eicosapentaenoate as the result of the binding of ${ }^{1} \mathrm{O}_{2}$ to the double bonds at the $\Delta 5,8,11,14$ and 17 positions. The difference in the isomeric compositions of the monohydroperoxides between the autoxidation and the ${ }^{1} \mathrm{O}_{2}$ oxidation was that the nonconjugated diene isomers, 6- and 17-isomers, were specific to ${ }^{1} \mathrm{O}_{2}$ oxidation in the peroxidation of methyl eicosapentaenoate. Furthermore, monohydro- peroxide was the only product of the ${ }^{1} \mathrm{O}_{2}$ oxidation (Fig. 4).

Methyl eicosapentaenoate contains five double bonds in the molecule. Consequently, methyl eicosapentaenoate was less stable on autoxidation and ${ }^{1} \mathrm{O}_{2}$ oxidation (Figs. 3 and 6). In particular, the autoxidation proceeded much faster than with the other unsaturated fatty acid methyl esters. The results indicate that methyl eicosapentaenoate is rapidly peroxidized for deterioration. Research on antioxidants may unravel how to protect against the peroxidation of methyl eicosapentaenoate.

Acknowledgment. The authors thank Dr. S. Kimura, Taiyo Fishery Co., Ltd. for the gift of the methyl eicosapentaenoate concentrate.

\section{REFERENCES}

1) E. N. Frankel, Prog. Lipid Res., 19, 1 (1980).

2) M. K. Logani and R. E. Davis, Lipids, 15, 485 (1980).

3) S. Matsushita, Eiyo to Shokuryo, 35, 375 (1982).

4) J. Dyerberg and H. O. Bang, Lancet, 2, 433 (1979).

5) J. A. Jakubowski and N. G. Ardlie, Thrombos. Res., 16, 205 (1979).

6) K. Fukuzumi, S. Ito and S. Nakanishi, Yukagaku, 12, 89 (1963).

7) A. C. Noble and W. W. Nawar, J. Am. Oil Chem. Soc., 48, 800 (1971).

8) A. C. Noble and W. W. Nawar, J. Am. Oil Chem. Soc., 52, 92 (1975).

9) K. R. Kopecky and H. J. Reich, Can. J. Biochem., 43, 2265 (1965).

10) D. A. White, "Form and Function of Phospholipids," ed. by G. B. Ansell, J. N. Hawthorne and R. M. C. Dawson, Elsevier, Amsterdam, 1973, p. 441.

11) J. Terao and S. Matsushita, Agric. Biol. Chem., 39, 2027 (1975).

12) R. Yamauchi, M. Kojima, K. Kato and Y. Ueno, Agric. Biol. Chem., in contribution.

13) K. Oette, J. Lipid Res., 6, 449 (1965).

14) K. Sohde, T. Ogawa and S. Matsushita, Yukagaku, 23, 228 (1974).

15) J. Terao and S. Matsushita, Agric. Biol. Chem., 45, 587 (1981).

16) H. W.-S. Chan and G. Levett, Lipids, 12, 99 (1977).

17) N. W.-S. Chan and G. Levett, Lipids, 12, 837 (1977).

18) E. N. Frankel, W. E. Neff, W. K. Rohwedder, B. P. S. Khambay, R. F. Garwood and B. C. L. Weedon, Lipids, 12, 901 (1977).

19) E. N. Frankel, W. E. Neff, W. K. Rohwedder, B. P. S. Khambay, R. F. Garwood and B. C. L. Weedon, Lipids, 12, 908 (1977). 
20) N. A. Porter, R. A. Wolf, E. M. Yarbro and H. Weenen, Biochem. Biophys. Res. Commun., 89, 1058 (1979).

21) W. A. Pryor, J. P. Stanley and E. Blair, Lipids, 11, 370 (1976).

22) E. N. Frankel, W. E. Neff, W. K. Rohwedder, B. P. S. Khambay, R. F. Garwood and B. C. L. Weedon, Lipids, 12, 1055 (1977).

23) W. E. Neff, E. N. Frankel and D. Weisleder, Lipids, 16, 439 (1981).

24) F. A. Kuehl, Jr. and R. W. Egan, Science, 210, 978
(1980).

25) R. J. Gryglewski, J. A. Salmon, F. B. Ubatuba, B. C. Weatherly, S. Moncada and J. R. Vane, Prostaglandins, 18, 453 (1979).

26) J. Terao and S. Matsushita, Agric. Biol. Chem., 45, 595 (1981).

27) H. R. Rawls and P. A. Van Santen, J. Am. Oil Chem. Soc., 47, 121 (1970).

28) W. E. Neff and E. N. Frankel, Lipids, 15, 587 (1980).

29) J. Terao and S. Matsushita, J. Food Process. Preserv., 3, 329 (1980). 